\title{
How Dickkopf molecules and Wnt/ $\beta$-catenin interplay to self-organise the Hydra body axis
}

\author{
Moritz Mercker ${ }^{1, *}$, Alexey Kazarnikov ${ }^{1,3}$, Anja Tursch ${ }^{2}$, Suat Özbek ${ }^{2}$, Thomas Holstein ${ }^{2}$, Anna \\ Marciniak-Czochra ${ }^{1}$ \\ 1 Institute of Applied Mathematics (IAM), BioQuant and Interdisciplinary Center of \\ Scientific Computing (IWR), Heidelberg University, Heidelberg, Germany \\ 2 Centre for Organismal Studies (COS), Heidelberg University, Heidelberg, Germany \\ 3 Southern Mathematical Institute of the Vladikavkaz Scientific Centre of the Russian \\ Academy of Sciences, Vladikavkaz, Russia \\ * E-mail: mmercker_bioscience@gmx.de
}

\begin{abstract}
The antagonising interplay between canonical Wnt signalling and Dickkopf (Dkk) molecules has been identified in various processes involved in tissue organisation, such as stem cell differentiation and bodyaxis formation. Disruption of the interplay between these molecules is related to several diseases in humans. However, the detailed molecular mechanisms of the $\beta$-catenin/Wnt-Dkk interplay leading to robust formation of the body axis remain elusive. Although the $\beta$-catenin/Wnt signalling system has been shown in the pre-bilaterian model organism Hydra to interact with two ancestral Dkks (HyDkk1/2/4$\mathrm{A}$ and $-\mathrm{C}$ ) to self-organise and regenerate the body axis, the observed Dkk expression patterns do not match any current pattern-formation theory, such as the famous activator-inhibitor model. To explore the function of Dkk in Hydra patterning process, we propose a new mathematical model which accounts for the two Dkks in interplay with HyWnt3/ $\beta$-catenin. Using a systematic numerical study, we demonstrate that the chosen set of interactions is sufficient to explain de novo body-axis gradient formation in Hydra. The presented mutual inhibition model goes beyond the classical activator-inhibitor model and shows that a molecular mechanism based on mutual inhibition may replace the local activation/long-range inhibition loop. The new model is validated using a range of perturbation experiments. It resolves several contradictions between previous models and experimental data, and provides an explanation for the interplay between injury response and pattern formation.
\end{abstract}

\section{Introduction}

Formation of the primary body axis is a fundamental step during embryogenesis, and the way in which this pattern is established remains a central but only partially answered question in biology $[1,2]$. An interplay between $\beta$-catenin and Wnt signalling appears to control posterior identity in various organisms, including pre-bilaterians and bilaterians, whereas anterior identity is conferred by their local inhibition, frequently realised by secreted Dickkopf (Dkk) proteins [2]. Dkks have been shown to antagonise posterior-related expression, e.g., through inhibition of the Lrp5/Lrp6-Wnt-Frizzled complex [3] or by direct reduction of $\beta$-catenin $[4,5]$. Local inhibition of canonical Wnt signalling by three of the four Dkk protein family members (Dkk1, Dkk2, and Dkk4) appears to be essential during vertebrate embryogenesis, particularly during body-axis and head formation, limb and eye patterning, and somitogenesis [6]. Notably, Dkk malfunction has been related to bone diseases, Alzheimer's disease, and cancer in humans [6,7].

The antagonistic interplay between Dkks and head-related $\beta$-catenin/Wnt molecules occurs in prebilaterians, such as the classical early metazoan model organism Hydra [8-10], where this interaction appears to be crucial for axis/head formation and regeneration [11-15]. In particular, the expression of nuclear $\beta$-catenin/T-cell factor (Tcf), as well as multiple Wnts (with HyWnt3 as one of the earliest ex- 
pressed Wnts), defines the oral pole [11-13,16], whereas HyDkk1/2/4-A and HyDkk1/2/4-C are expressed in gland cells within the body column below the head, antagonising Wnt/ $\beta$-catenin signalling $[14,15]$. The vertebrate Dkk1, Dkk2, and Dkk4 may have developed from these HyDkk1/2/4 homologues by gene duplication [14].

Strikingly, both HyDkk1/2/4 genes are located on the same genomic scaffold in Hydra magnipapillata (Sc4wPfr_134). The last exon of Dkk1/2/4-A is only $4 k b$ upstream of the first exon of HyDkk1/2/4-C, indicating that HyDkk1/2/4-C probably arose from HyDkk1/2/4-A by gene duplication. Both HyDkk1/2/4 genes of Hydra are unique in having only one CRD from the two CRD motifs found in bilaterian Dkks (CRD-1 and -2). Moreover, the CRD of both HyDkk1/2/4 genes shares a sequence similarity of $78 \%$ on the protein level. Phylogenetic analyses revealed that both, HyDkk1/2/4-A and -C, bear the closest similarity to the CRD-2 motif of vertebrate Dkk genes 1,2, and 4; the domain that exclusively determines Wnt-antagonising activity [6]. Yet, while the inhibitory function on canonical Wnt signalling has been already demonstrated for HyDkk1/2/4-A in Xenopus, the direct experimental evidence for a Wntantagonising effect of HyDkk1/2/4C is still pending [14,15]. The only known Hydra Dkk members that comprise both, a regulatory CRD-1 and executive CRD-2, belong to the Dkk3 family. In contrast to HyDkk1/2/4-related molecules, HyDkk3 does not antagonise canonical Wnt signalling in bilaterians [17]. Evidence suggests that HyDkk3 is involved in tentacle development (Özbek, unpublished) and/or nematogenesis [18] in Hydra, rather than in axis/head formation. However, HyDkk3 has been proposed to represents the ancestral Dkk type, from which HyDkk1/2/4 may have developed by gene duplication, thereby probably paving the way for the evolution of vertebrate Dkk1, Dkk2, and Dkk4 by further duplication events $[14,18]$.

Body-axis formation in Hydra is a self-organised process, and functional polyps can regenerate from a re-aggregated bulk of dissociated and randomly mixed cells ("Hydra aggregates") [19,20]. This process is considered to represent de novo pattern formation, because cells differentiate rather than re-sort based on their different axial origins [21,22]. Various attempts have been made to explain de novo pattern formation based on simplified mathematical models. A general mathematical theory proposed by Alan Turing [23] linked the symmetry-breaking phenomenon with the diffusive instability of a spatially homogeneous state. In the context of Hydra, the Turing idea has been elaborated in the seminal "activator-inhibitor" model of Gierer and Meinhardt [24-26], which is a system of nonlinear reactiondiffusion equations describing the interplay between "local activation and long-range inhibition" (LALI) of two diffusing morphogen species $[25,27]$. Later, this model has been augmented by a third component, the 'source density' (SD), which is a rather slowly changing long-term storage of the body axis gradient $[26,28]$. Although the activator-inhibitor model can explain several aspects of de novo pattern formation, the model remains controversial, and while Wnt signalling can be linked to the hypothetical activator, no inhibitor that satisfies the assumptions of the model has yet been identified at the molecular level. Various alternative models taking account of more diffusive biochemical interactions $[29,30]$, and coupling of diffusive signalling factors with non-diffusive biochemical networks [31-34] or with mechanical signal transduction [35-37] have been shown to exhibit de novo pattern formation. The different models show diverse biophysical processes that may lead to LALI-based self-organisation, including mechanical or mechano-chemical, cellular, and bio-electrical processes [27,35-39]. Given the potential richness of these underlying mechanisms, it is of limited use to search for molecular candidates to fit specific existing/proposed pattern-formation models which have not been developed strictly based on experimental data.

Indeed, the experimentally observed expression patterns of Dkk in Hydra do not match any of the above models. In particular, HyDkk1/2/4-A is uniformly expressed throughout the entire body column outside the head, whereas HyDkk1/2/4-C expression is fading out in the aboral direction $[14,15]$. 
Expression of both molecules is thus lacking at the oral pole, whereas the activator-inhibitor model assumes that the expression pattern of the head activator and its antagonising molecule coincide $[24,25]$. Interestingly, the expression patterns of HyDkk1/2/4-A resemble that of a "substrate molecule" in the activator-depleted substrate model, a second type of model proposed by Gierer and Meinhardt [25]. However, this model requires that HyDkk1/2/4-A activates head-related molecules (such as canonical Wnt signalling), but rather the contrary is suggested from molecular knowledge [6] and functional studies in Hydra [40]. Additionally, expression of both Dkks appears to be downregulated after pharmacological GSK3 inhibition [14,15]. All these observations have led to confusion regarding the role of Dkks in Hydra pattern formation, and these molecules have consequently been ruled out as possible candidates fitting the current theory of self-organised pattern formation $[26,41]$. Recent experimental searches for the molecular components of the de novo patterning system have instead focused on the missing inhibitor postulated by the activator-inhibitor model [42].

Attention has turned to the transcription factor Sp5 [42], which is a Wnt target and negatively regulates Wnt transcription in human pluripotent stem cells [43]. However, Sp5 does not fit all assumptions of the activator-inhibitor model, given that it is probably non-diffusive, but strong diffusion is required for the inhibitor of the activator-inhibitor model. Furthermore, during homeostasis, experimentally observed Sp5 patterns differ from the expected pattern for an inhibitor since Sp5 expression is missing in the outermost head, where the model assumes maximal expression. Several results on Sp5 in Hydra [42] indicate that Sp5 is involved in the HyWnt3-driven small-scale pattern formation of the head organiser, rather than in establishing the large-scale body-axis gradient [44]. The extracellular matrix molecule thrombospondin (TSP) has also been reported to antagonize $\beta$-catenin/Wnt signalling in Hydra [45], but its expression patterns also differed from those predicted by the previous pattern formation theories: TSP is expressed not only at the oral pole but also at the base of the tentacles, indicating a general or versatile rather than a head-specific role during chemical and/or mechanical tissue patterning in Hydra. Furthermore, experimental manipulations of TSP levels have no apparent influence on the number of axes/heads, and only affect the number of ectopic tentacles after activation of $\beta$-catenin/Wnt signalling [45]. If and how TSP is involved in head and/or tentacle formation thus remains unclear. Recently, an astacin family proteinase (HAS-7) has been identified, processing HyWnt3 and thus most probably contributing to head inhibition [40]. Strikingly, HAS-7 shows several parallels to HyDkk1/2/4-C: not only in situ expression patterns are very similar, but also assumed qualitative interactions with the canonical Wnt signalling complex show several parallels - although the molecular inhibition mechanisms are different (Wnt degradation vs. co-receptor isolation).

In summary, although antagonistic interactions between $\beta$-catenin/Wnt and Dkks have been frequently observed during axis formation in Hydra, suggesting an early evolutionary role during pattern formation, how the molecular interactions between these players lead to spontaneous formation of the body axis is still unknown. In particular, Dkk-related experimental observations (such as expression patterns) contradict previous pattern-formation theories, and these issues cannot be resolved by other molecular candidates antagonising head and axis formation in Hydra.

Instead of searching for new molecular candidates to fit the existing pattern-formation models, we propose a new mathematical model based on interactions between $\beta$-catenin/HyWnt3 and Dkks. The model is named "mutual inhibition model" after its core feedback loop that stands behind the pattern forming mechanism. The aim of this bottom-up approach is to determine if a known structure of a signalling network is sufficient to explain de novo formation of the body axis in Hydra. The choice of model components and their interactions is motivated by their documented roles during Hydra axis formation and regeneration [11-15]. The interplay between canonical Wnt signalling and Dkks appears to play a major developmental role in several higher animals (including vertebrates) [6], suggesting an evolutionary 


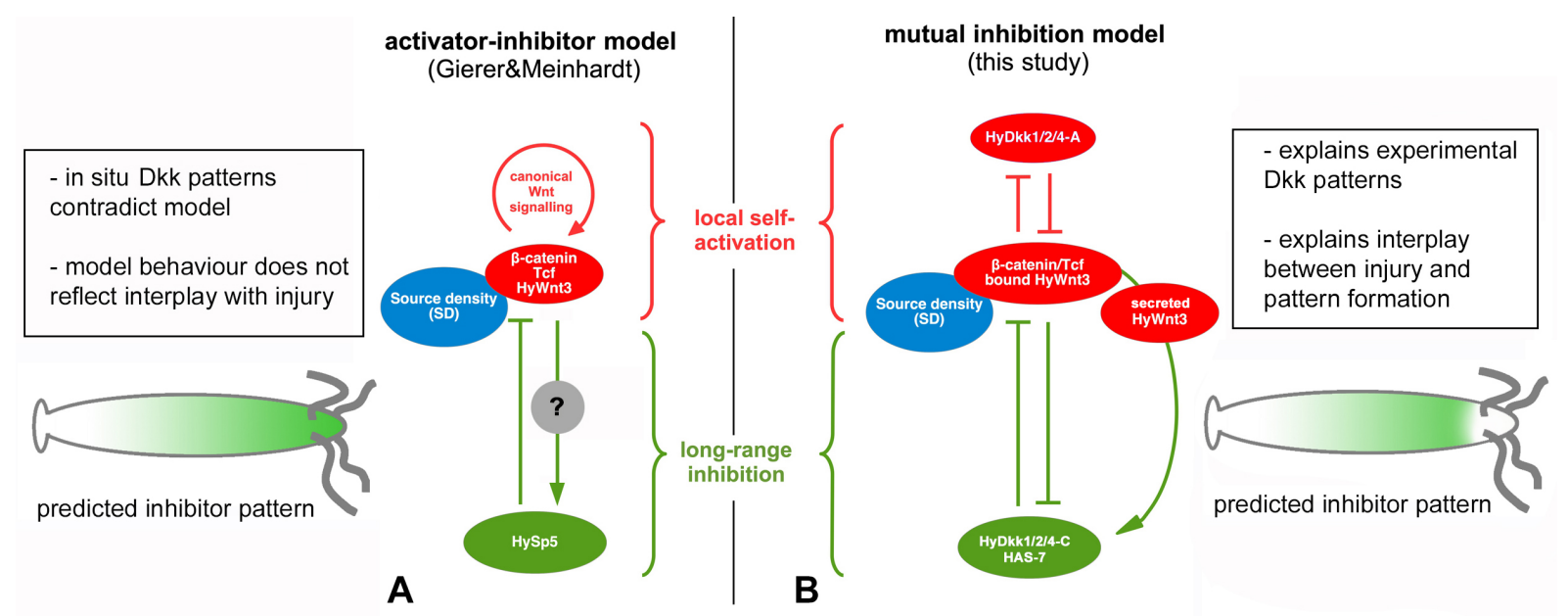

Figure 1. Schematic comparison of the activator inhibitor model of Gierer \& Meinhardt [26] (A) compared to the mutual inhibition model as proposed in this study (B). Both models satisfy the general principle of local self activation (red) and long-range inhibition (green), and additionally interplay with the source density (blue). Grey circles with interrogation marks represent still unknown long-ranging mechanisms. Model limitations respectively improvements are listed within grey boxes.

Self-amplification due to canonical Wnt signalling is not explicitly considered in the mutual inhibition model for the sake of complexity, but may depict an additional/redundant mechanism for local self activation. Predicted expression patterns of the inhibitor differ between the models.

highly conserved process and thus an interesting mechanism.

We used mathematical modelling and simulations to demonstrate that qualitative interactions among $\beta$-catenin/Wnt signalling and the two Dkk-molecules HyDkk1/2/4-A and HyDkk1/2/4-C, and the longterm storage of the body-axis gradient (source density) were sufficient to spontaneously create the Hydra body axis. Here, the recently described Wnt protease HAS-7 [40] fulfills the same criteria as Dkk1/2/4-C and could therefore replace/augment the function of the latter in the proposed model. Although the presented model relies on the Turing-type mechanism of symmetry breaking which is a general principle behind the classical LALI models, the molecular realisation is distinct different from the scheme assumed in the activator-inhibitor model (c.f., Fig. 1). Where the previous models of Gierer and Meinhardt aimed to formulate the LALI principle in the most intuitive way, in this work, we demonstrate that experimental data suggest a different way of molecular realisation, which is based on a tight interplay between canonical Wnt signalling and different Dkk molecules (and/or proteases) with negative feedback loops playing a prominent role (Fig. 1). We validated the model by showing that simulations of various perturbation experiments produced experimentally observed expression patterns. We further demonstrated that this new pattern-formation model exhibited different dynamics from the activator-inhibitor model, in accordance with several experimental observations, such as the interplay with local injury signalling. Generally, this study demonstrates the benefit of investigating the ability of a known network of molecular interactions to generate experimentally observed patterns, rather than searching for molecules to fit a specific existing pattern-formation theory, such as the activator-inhibitor model. 

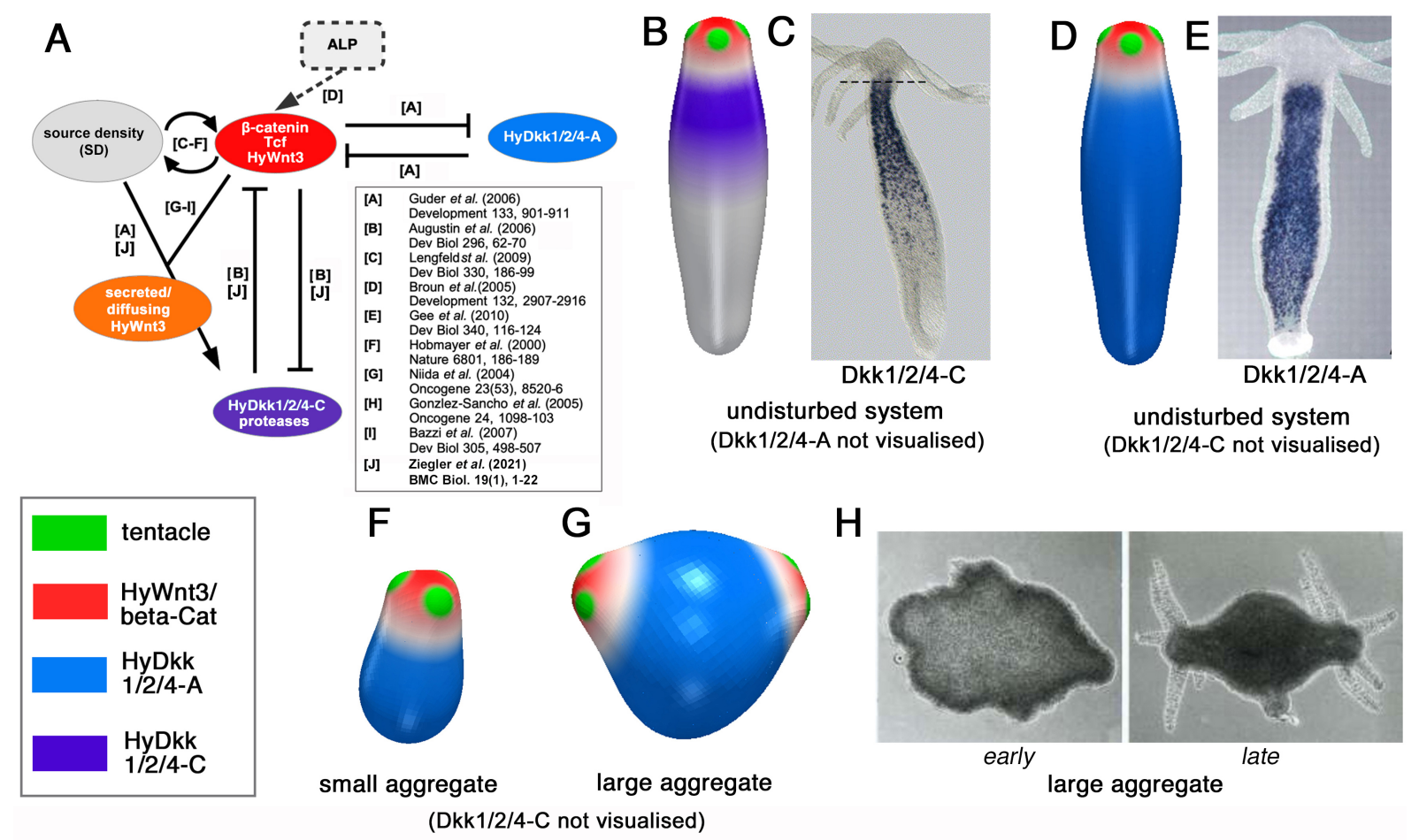

Figure 2. (A) Sketch of the Hydra body-axis formation network including main references. For the sake of clarity, interactions with the tentacle and foot system are not shown. (B) Simulated (purple) and $(\mathrm{C})$ experimentally observed expression patterns of HyDkk1/2/4-C in wild-type; (D) simulated (blue) and (E) experimentally observed expression patterns for HyDkk1/2/4-A in wild-type. (F)-(G) late stages of simulated small and large aggregate; HyDkk1/2/4-C expression is not visualised in (F)-(G) for the sake of clarity. $(\mathrm{H})$ Early and late stages of experimental large aggregate. Colour-scaling is similar in all simulation snapshots in this figure. (C) is taken from Ref. [15], (E) from Ref. [14].

\section{Results/Discussion}

An overview of the detailed simulated molecular interaction scheme derived from various experiments is given in Fig. 2 A. In particular, the considered Hydra body-axis pattern formation system (only) comprised the interactions among the $\beta$-catenin/HyWnt3 complex, the slowly changing source density, and the two Dkk molecules HyDkk1/2/4-A and HyDkk1/2/4-C (an alternative/additional molecular candidate for the latter is HAS-7). Additional interactions with the tentacle- and foot-pattern formation system were not the focus of the study and are therefore not shown in the scheme for the sake of clarity; however detailed corresponding information and simulations are given in e.g. Ref. [26, 28, 44]. More information on the Wnt/Dkk part of the model, including the detailed experimental motivations for all the presented interactions, are given in the Materials and Methods section, and in the references depicted in Fig. 2 A.

Based on the above-mentioned interaction network, we first simulated head regeneration in the undisturbed (wild-type) system by providing an initial geometric and chemical body-axis gradient (the latter given by a graded SD), with all other molecules initially randomly distributed. We observed regeneration of the head, with the final patterns strongly resembling experimental observations (Fig. 2 B-E). Notably, 
both HyDkk1/2/4-related molecules were lacking in the hypostome, showing a sharp expression border beneath the tentacles, where HyDkk1/2/4-C expression faded out in the aboral direction (Fig. 2 B-C), in contrast to HyDkk1/2/4-A which was strongly expressed throughout the entire body column (Fig. 2 D-E). This model thus completely resolved the previous contradiction between Dkk expression patterns and the activator-inhibitor model $[26,42]$.

In the stable pattern resulting from head regeneration simulations, the $\beta$-catenin/HyWnt3 complex appeared to be restricted to the upper head, fading within and below the tentacle zone (Fig. 2 B,D). The latter expression patterns strongly resembled the experimentally observed patterns for $\beta$-catenin in adult Hydra [11,46], in contrast to the always smaller and sharper HyWnt3 expression patches observed in experiments (e.g., $[11,42]$ ). It should be emphasised that $\beta$-catenin is always an intracellular factor that potentially interacts with the machinery of transcriptional control (Tcf, Groucho, HDAC), whereas Wnts are extracellular factors secreted by the epithelial cells. Thus, the graded distribution of $\beta$-catenin is always the result of the activity of signal transduction from a diffusible factor received by the target cells (such as Wnt in canonical Wnt signalling). As indicated in the Materials and Methods section, we condensed the known cell-local molecules associated with head activation in Hydra into a single virtual component in the current simulation model, to minimize model complexity. Various experiments, in conjunction with simulation studies, indicated that $\beta$-catenin/Tcf vs. HyWnt3 were likely to contribute to two separate de novo pattern-formation systems in Hydra, organising development and differentiation at two different spatial scales [44]. In particular, nuclear $\beta$-catenin appears to act at the scale of the body and plays a role in body-axis formation, whereas canonical HyWnt3 signalling is involved in a downstream pathway responsible for small-scale patterning of the head organiser, eventually controlling head formation [44]. The condensed $\beta$-catenin/Tcf/HyWnt3 complex considered within our model was thus likely related to cell-local $\beta$-catenin/Tcf, which may in the real system again locally induce a small patch of an HyWnt3-expressing organiser to induce and control small-scale patterning during head formation.

The difference in expression patterns between the two Dkk molecules in the body column is a result of qualitative differences in the corresponding production-terms (for corresponding experiental evidence, c.f., section "Submodel for self-organised body-axis formation"): where HyDkk1/2/4-A is assumed to be constantly produced in the absence of repressing signals, HyDkk1/2/4-C (and/or HAS-7) production is downstream of the SD respectively $\beta$-catenin/HyWnt3 and thus fades out in aboral direction. It is important to notice that although both molecules show overlapping expression domains, HyDkk1/2/4-A fulfils the role of local-activation, whereas HyDkk1/2/4-C is rather involved in long-range inhibition (c.f., Fig. 1 and Fig. 2 A). This apparent contradiction between antagonistic activity and similar expression patterns arises from the fact that local activation is realised through the inhibition of the inhibitor HyDkk1/2/4-A. Thus, the inverse expression pattern of HyDkk1/2/4-A is approximately comparable to the activator pattern in the activator-inhibitor model.

To investigate if the observed ability of self-organised axis formation critically dependeds on the initially provided geometric or chemical (SD) gradient, we then simulated Hydra aggregates, i.e., starting the simulations with a symmetric tissue sphere and a random distribution of all considered chemical components, including the SD. Indeed, the simulated system developed de novo patterns related to the experimentally observed patterns in adult polyps. Notably, small initial spheres developed into a single polyp (Fig. $2 \mathrm{~F}$ ), and larger simulated systems developed multiple heads (Fig. $2 \mathrm{G}$ ), similar to the experimental observations (Fig. $2 \mathrm{H}$ ).

The scheme presented in Fig. 1 (respectively Fig. 2 A) thus represents a true de novo patternformation system, which appears to be distinctly different from previously described pattern formation systems, such as the activator-inhibitor model or the activator-depleted substrate model [24, 25]. 
This difference concerns various aspects of the model. First, the main interactions (namely between $\beta$-catenin/HyWnt3 and the HyDkk1/2/4-A and -C) consisted only of mutual inhibition, with no direct self-activation loop. Indeed, such mutual local repressions increasingly appear to be an important developmental tool guaranteeing robust patterning of the body axis in higher organisms [47]. Second, mutual repression at the molecular scale resulted in a lack of Dkk expression in the hypostome, which previously led to the conclusion that Hydra Dkks could not serve as long-range inhibitors in the context of the activatorinhibitor model $[26,42]$. However, although both Dkks did not meet the requirements proposed by the activator-inhibitor model, they nevertheless realised a more general principle required for self-organised patterning, namely the LALI principle [25, 27,41]. Indeed, mutual repression of $\beta$-catenin/HyWnt3 vs. HyDkk1/2/4-A effectively led to local self-activation (via the inhibition of an inhibition), as a mechanism proposed to drive local self-activation in other patterning systems [41]. In contrast, HyDkk1/2/4-C (and similarly HAS-7 [40]) appeared to realise long-range inhibition, being additionally activated by the SD respectively the secreted HyWnt3 molecules (possibly including additional fast-diffusing factors [40]) to achieve the long-range effect. Also here, the additional local inhibition from $\beta$-catenin/HyWnt3 to HyDkk1/2/4-C (respectively HAS-7) leads to a lack of Dkk in the head - in contrast to the maximal expression of the inhibitor proposed by the activator-inhibitor model [25]. Without this mutual negative feedback between $\beta$-catenin/HyWnt3 tand HyDkk1/2/4-C, the levels of the latter would be greatest at the Hydra head, as observed for the inhibitor in the classical activator-inhibitor model. However, the additional repression adds robustness to the final pattern, given that local self-activation is increased due to the additional elimination of HyDkk1/2/4-C from the upper head. Third, it appears that the presented model (in contrast to the activator-inhibitor model) requires an initial perturbation of a certain strength in order to initiate pattern formation - which matches well experimental observations concerning the interplay of injury response with patterning. More details on this subject are given below.

In summary, the general LALI principle is fulfilled by the proposed model and the investigated interactions among $\beta$-catenin/HyWnt3, the SD, and the two Dkk molecules. The realisation however differs from previous models, such as the activator-inhibitor or activator-depleted substrate model [25]. This is in line with the more general observation that various chemical networks can spontaneously create patterns $[29,30]$, and the previously proposed models (such as the activator-inhibitor model) are thus only special cases of a rich selection of possible networks leading to self-organisation [27]. It is thus unlikely that real patterning networks correspond exactly to these models, and instead of looking for appropriate molecular candidates to fit known/previous models, researchers should rather investigate the pattern-formation potential of experimentally well-characterised networks.

The current new model is in accordance with both experimentally observed qualitative interactions between different molecules, and with experimentally observed expression patterns of the two Dkk molecules. In addition, it also resolves further contradictions between the activator-inhibitor model and previous experiments, in which the activator-inhibitor is highly unstable, such that even small perturbations from homogeneously distributed molecules can lead to self-organised pattern formation. In contrast, various experiments with Hydra showed that head/axis patterning only occurred if the system was strongly perturbed, reflecting the ability of the animal to control the patterning process. Bud formation (which is basically the formation of an additional head/axis) only occurs under specific environmental conditions [48], and a certain level of $\beta$-catenin/HyWnt3 is required to induce head or foot formation and regeneration [20,49], while an injury-related initial increase in developmental genes appears to be required to induce proper head regeneration [14,49,50] and can additionally increase the frequency of budding [51]. Indeed, the ability of the current new model to form de novo patterns appears to critically depend on the initial distribution of the $\mathrm{SD}$ respectively $\beta$-catenin/HyWnt3: if either initial $\mathrm{SD} / \beta$-catein/HyWnt3 values fell below a certain threshold during the simulation of Hydra aggregates, or the stochastic perturbation of $\mathrm{SD} / \beta$-catein/HyWnt3 is too weak, the system lost its ability to form patterns and converged instead 


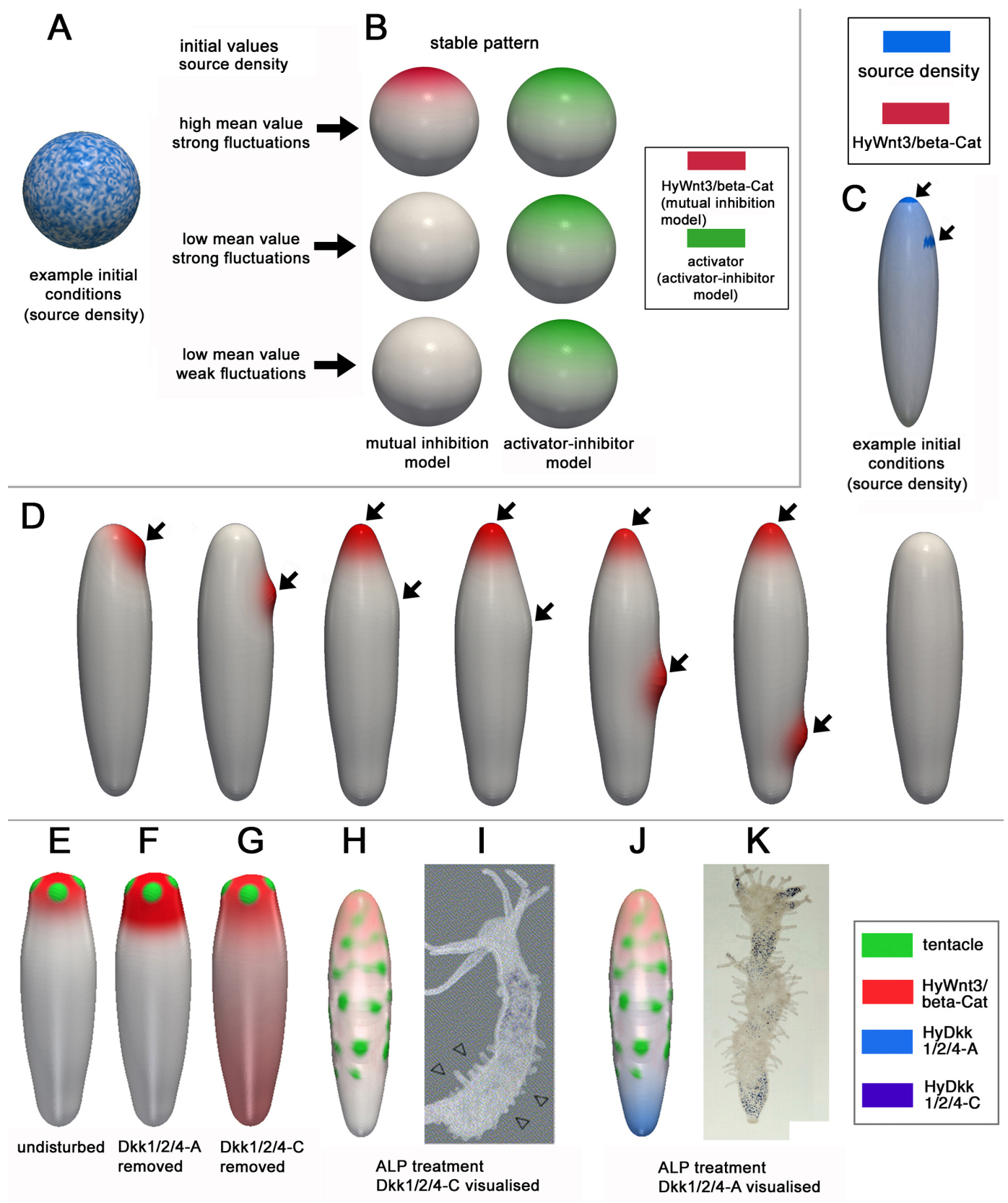

Figure 3. Simulations of regeneration in Hydra aggregates $(\mathrm{A}, \mathrm{B})$ and of regeneration/grafting in Hydra polyps (C,D). A: example aggregate initial conditions, B: different observed stable patterns, C: example regeneration/grafting initial conditions, D: different resulting patterns. In the mutual inhibition model, the ability to form de novo patterns depends on both: initial mean values as well as fluctuation strength - in contrast to the activator-inhibitor model, the latter always forming patterns (A-B). During simulated grafting, two heads can only develop if initial grafts (defined by initial high SD values, location is indicated by black arrows) are sufficiently distant to each other (C-D). Simulated $\beta$-catenin/HyWnt3 expression (red) in wild-type (E), after removing HyDkk1/2/4-A (F) and HyDkk1/2/4-C (G). (H) Simulated (purple) and (I) experimentally observed expression patterns of HyDkk1/2/4-C after ALP treatment; (J) simulated (blue) and (K) experimentally observed expression patterns of HyDkk1/2/4-A after ALP treatment. (I) is taken from Ref. [15], (K) from Ref. [14]. 


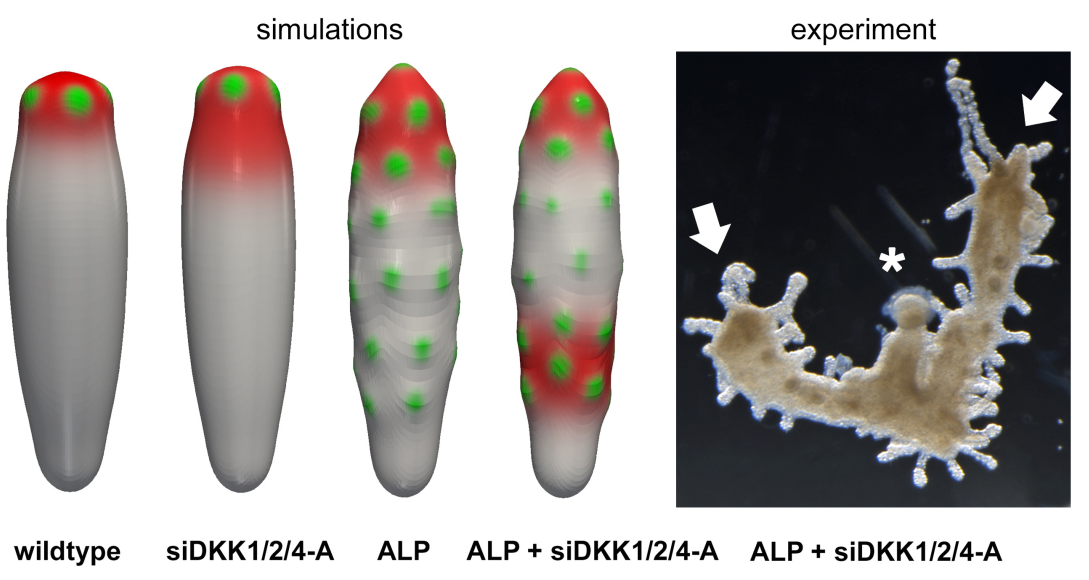

Figure 4. Left side: simulation snapshots of four different virtual experiments investigating the effects of ALP treatment and/or HyDkk1/2/4-A-knockdown on simulated $\beta$-catenin/HyWnt3 expression (red) and tentacles (green). Right side: ALP plus HyDkk1/2/4-A-knockdown experiment (arrows: head, asterisk: foot/peduncle).

on a homogeneous state, where all molecules showed low constant or zero expression. The activatorinhibitor model, in contrast, forms patterns in all considered scenarios (Fig. 3 A,B). The same holds for the head regeneration process: if the initial SD gradient is chosen such, that it's maximum is below the maximal value of the steady state head structure, an additional initial $\mathrm{SD} / \beta$-catenin/HyWnt3 signal is required to initiate head regeneration (Fig. $3 \mathrm{C}, \mathrm{D}$ ). Mathematically, the observed patterns in the new model most likely belonged to the class of "far-from-equilibrium patterns" [33,34], in contrast to the "close-to-equilibrium patterns" usually observed/analysed in the context of the activator-inhibitor model.

Further, we simulated various regeneration and transplantation experiments, depicting classical and seminal experiments leading among others to the discovery of the head organiser [52] and the discovery of distinct antagonistic gradients of head activation and inhibition potentials [53,54]. The simulations revealed that the mutual inhibition model reproduces all key properties of the real system, namely (1) the necessity of an initial strong local $\beta$-catenin/HyWnt3/SD signal in order to initiate axis formation (e.g., induced by an injury or a high head activation potential/source density within the tissue piece from the oral tip); (2) the observation of head inhibition (if two initiating signals are too close to each other only one head develops); and (3) bud formation can only be initiated within the budding zone - if simultaneously a head exists/regenerates at the oral pole. Without the head at the oral pole, grafts can induce a secondary axis at arbitrary locations (Fig. 3 C,D). All these observed properties result from the interplay between all simulated components in the system, and thus cannot be attributed to a single component in the system.

To further validate the proposed model, we repeated several key experiments virtually with respect to the discovery of the two HyDkk1/2/4-related molecules in Hydra Ref. [14,15]. First, we performed simulations of the undisturbed system until the head patterns stabilised (related to Fig. 2 B,D and $3 \mathrm{E}$ ), and subsequently removed HyDkk1/2/4-A and HyDkk1/2/4-C expression, respectively, from the simulated system. In accordance with experimental observations, we observed distinct expansion of the $\beta$-catenin/HyWnt3-expression zone in the head after removing HyDkk1/2/4-A (Fig. $3 \mathrm{~F}$ and Ref. [14]) and body-wide ectopic expression of $\beta$-catenin/HyWnt3 after removing HyDkk1/2/4-C (Fig. $3 \mathrm{G}$ and Ref. [15]). The only difference between the simulations and experiments was that $\beta$-catenin/HyWnt3 ex- 
pression after reducing the number of HyDkk1/2/4-C-expressing cells was relatively diffuse/homogeneous (Fig. $3 \mathrm{G}$ ), whereas ectopic spots of HyWnt3 expression were experimentally observed [15]. This may also be related to the fact that cell-local $\beta$-catenin and HyWnt3 were condensed as one virtual component in the simulated model, whereas they may actually be connected to two different pattern-formation systems acting at two different spatial scales [44]. Second, we simulated activation of canonical Wnt signalling via ALP treatment (Fig. $3 \mathrm{H}, \mathrm{J}$ ). Experimentally, both Dkks appear to be suppressed by this treatment, along with the development of ectopic tentacles all over the body column [14, 15]. However, HyDkk1/2/4-C expression seem to react more sensitively to $\beta$-catenin/HyWnt3-levels, since HyDkk1/2/4-A reduction appears to be later than HyDkk1/2/4-C reduction [14,15]. These observations are in well accordance with our simulation results at the moment of ectopic tentacle development (Fig. $3 \mathrm{H}-\mathrm{K}$ ). However, we want to point out that in late stages of these simulations, Dickkopf patterns re-establish, which might reflect the fact that terminal cell differentiation is not incorporated in our model and ALP-driven effects are only initially given and thus reversible. Finally, we also repeated recent HyDkk1/2/4-A-knockdown experiments [40] with and without ALP treatment (Fig. 4). Similar to experimental results, combined ALP treatment and HyDkk1/2/4-A knockdown resulted in the development of a secondary ectopic axis (give by an additional region with strong expressio of the canonical Wnt signalling complex).

The experimental observation that interstitial cells (precursors of the Dkk-expressing gland cells) are not required for regeneration [55-57] may question if Dkk is indeed essential for Hydra pattern formation. However, epithelial- and interstitial-derived cells showed high plasticity in Hydra [58] (e.g. neurogenic activity can be compensated for by epithelial cells when neurons are missing [59]), and similar mechanisms could adopt Dkk expression after the removal of interstitial cells. Furthermore, Dkk knockdown [40] or removal of gland cells [14,15] demonstrate distinct perturbation of pattern formation in Hydra. Finally, the Dkk-system may represent only one of different partially redundant pattern formation systems in Hydra to ensure robustness (c.f., below).

\section{Summary and Outlook}

In this study, we demonstrated that de novo axis formation in Hydra can be explained by known qualitative interactions between head-related molecules, such as $\beta$-catenin/Tcf/HyWnt3, HyDkk1/2/4-A and HyDkk1/2/4-C (and/or HAS-7), and the source density. In particular, and based on various experimental data in conjunction with new simulation studies, we demonstrate that HyDkk1/2/4-A and HyDkk1/2/4$\mathrm{C}$ in interplay with canonical Wnt signalling and the source density such that the famous principle of local activation/long-range inhibition [25,27] is fulfilled. However, the molecular realisation is different (and less intuitive) compared to the assumed network in the classical activator-inhibitor model.

Notably, we demonstrated that this system could explain steady-state Dkk expression patterns in adult polyps, self-organised head regeneration, as well as the regeneration of the entire body axis from initially randomly mixed cells. Additional validation was obtained by performing several key experiments virtually (ranging from classical regeneration and grafting experiments up to recent molecular manipulation studies), and demonstrating that the resulting patterns matched the corresponding experimental observations. Although the investigated model fulfils some of the general principles required for selforganisation, the considered system has various distinct qualitative differences compared with previous patterning models, in good accordance with experimental observations, such as the requirement for sufficiently strong initial expression in order to initiate head formation and regeneration $[14,49,50]$. The presented de novo patterning mechanism could be conserved across multiple taxa, given that canonical Wnt signalling in interplay with Dkks appears to be a crucial driving force during axis formation in various organisms [6]. 
We want to point out that the presented mechanism may depict only one of several partially redundant pattern formation mechanisms in Hydra, ensuring additional robustness of axis and head formation/regeneration. For example, canonical Wnt signalling (for the sake of complexity not explicitly modelled in the presented work) is assumed to be an additional mechanism for local activation [60], and Sp5 is a promising molecule in the context of an activator-inhibitor-type network [42], possibly required for small-scale organiser pattern formation [44]. Finally, head excision without (strong) injury response does not completely prevent but rather delays head regeneration (Holstein, unpublished), which could indicate the existence of both mechanisms - the mutual inhibition mechanism and an activator-inhibitortype network - acting in parallel.

At a more general level, this study demonstrated that self-organising networks may differ from (and possibly being less intuitive than) known pattern-formation models, such as the famous activator-inhibitor model [24-26], as recently indicated in studies demonstrating the potential richness of different chemical networks capable of self-organising into Turing-type patterns [29,30]. We suggest that, instead of searching for appropriate candidates to fulfil the predicted properties of known models, future pattern-formation research should focus on using in silico and/or mathematical techniques to investigate the capacities of known networks to explain experimentally observed patterns, even if these networks and/or expression patterns differ from those expected based on previously proposed/known models.

\section{Materials and Methods}

\section{Introduction of the mathematical model and its biological justification}

We developed a mathematical model focussing on the interactions between two Dkks and Wnt/ $\beta$-catenin signalling, augmented by long-term storage of the body-axis gradient (SD). We aimed to demonstrate that these interactions are sufficient to govern formation of the Hydra body axis. Additionally, we coupled this body-axis system with two separate pattern-formation systems controlling foot and tentacle formation. The resulting model of spatio-temporal signalling was simulated on a domain representing Hydra tissue, as a thin tissue for which the evolution was modelled using a geometric partial differential equation. The coupling of two submodels was provided by morphogen-dependent evolution of the tissue curvature. Importantly, neither the tentacle and foot system itself, nor the coupling to the tissue curvature directly influenced the de novo axis-formation process. These model ingredients were taken into account to allow additional model validation, e.g. using alsterpaullone (ALP) experiments, and to mimic realistic tissue geometry (a cigar-shaped body exhibiting local tissue evagination at developing tentacles). Although the proposed axis-formation model mainly consisted of lateral chemical reactions within the tissue surface, the geometry of the surface may indirectly influence the dynamics of the pattern-formation process [61,62]. Considering a realistic geometry and its evolution, we excluded such effects. Although the detailed molecular mechanisms responsible for foot and tentacle patterning in Hydra are still not known, they are phenomenologically well-described by activator-inhibitor-type models. The qualitative model of the head, foot, tentacle system and the SD has been experimentally justified and has been thoroughly discussed e.g. in Ref. [26,28]. The main focus of the current model was thus to test the de novo pattern-formation capacity of a model based on experimentally documented non-activator-inhibitor-type interactions of Wnt/ $\beta$-catenin signalling with HyDkk1/2/4-A and HyDkk1/2/4-C.

\section{Submodel for self-organised body-axis formation}

Previous early grafting and regeneration experiments demonstrated that Hydra patterning comprises two separate mechanisms acting at two different temporal scales, referred to as "stable" vs. "labile" head activation [53]. Indeed, following ALP treatment (blocking glycogen synthase kinase $3 \beta$ and thus increasing 
nuclear $\beta$-catenin [13]), the half-life of activated molecules (such as $\beta$-catenin, HyWnt3, or Brachyury) was in the range of hours up to a maximum of 2-3 days, whereas the body-axis gradient information lasted up to $6-8$ days $[12,26,63,64]$. Although its molecular/physical nature remains unknown, the existence of this long-term storage of body-axis gradient information has been experimentally demonstrated, and this property is referred by various terms, including "positional value" and "source density", see Refs. $[8,20,24,53,65-67]$. Following [26], we refer to this as source density (SD). Various experiments have demonstrated that SD is induced by high levels of HyWnt3/nuclear $\beta$-catenin $[12,13]$. In turn, head regeneration (respectively the HyWnt3/ $\beta$-catenin-mediated expression of head-related genes) always occurs at the most apical part of the Hydra body column $[11,16,64,68]$, indicating mutual activation between HyWnt $3 / \beta$-catenin signalling and the SD.

We recently demonstrated, using model-based analysis of experimental studies, that patterns of nuclear $\beta$-catenin and HyWnt3 in Hydra were controlled separately, organising development and differentiation at two different spatial scales [44]. Notably, our results suggested that nuclear $\beta$-catenin acted at the scale of the whole body to control axis formation, whereas canonical HyWnt3 signalling was involved in a downstream pathway responsible for small-scale patterning of the head [44]. The underlying model was based on hypothetical inhibitors. In the following, we show how interactions with Dkks may replace the hypothetical model components. To streamline the model presentation, we condensed cell-local $\beta$-catenin and HyWnt3 into a single model variable representing different cell-local head-related molecules (mainly belonging to the canonical Wnt signalling complex) and providing a basis for the "labile head activation" during axis/head formation [53].

Based on ALP treatment, Guder et al. [14] demonstrated that HyDkk1/2/4-A expression in Hydra was negatively regulated by HyWnt $3 / \beta$-catenin. Furthermore, they showed that this negative influence was mutual, given that removal of HyDkk1/2/4-A-expressing gland cells led to ectopic HyWnt3-expressing cell clusters throughout the body column. This inhibitory influence was additionally confirmed by analysis of the impact of Hydra HyDkk1/2/4-A on canonical Wnt signalling in Xenopus [14], and in another recent study showing that knockdown of HyDkk1/2/4-A in combination with ALP treatment led to the formation of ectopic axes in Hydra [40].

A similar mutual negative interplay has been suggested between HyDkk1/2/4-C and HyWnt3/ $\beta$ catenin. ALP treatment induced a strong body-wide reduction in HyDkk1/2/4-C expression, and removal of HyDkk1/2/4-C-expressing gland cells led to an extension of HyWnt3 expression in the head region [15]. However, the SD has demonstrated an additional positive influence on HyDkk1/2/4-C expression based on both co-expression studies and $\mathrm{LiCl}$ treatment [15], of which the latter is known to manipulate the $\mathrm{SD}$ in Hydra [66,69]. In contrast, HyDkk1/2/4-A expression levels were unchanged by manipulating the SD [15]. Thus, both HyDkk1/2/4-A and HyDkk1/2/4-C form part of a negative feedback loop with head-related "labile head-activation molecules" (such as $\beta$-catenin and HyWnt3), with HyDkk1/2/4-C being additionally positively influenced by the slowly changing SD.

The observed ring pattern of HyDkk1/2/4-C expression suggests that the Wnt-related activation of HyDkk1/2/4-C and its inhibition must act on different space scales. Thus, we assume a positive influence from secreted HyWnt3 on HyDkk1/2/4-C expression. This assumption is additionally supported by the experimental evidence in other organisms showing activation of different Dkks by molecules from the canonical Wnt signalling pathway [70-72]. The assumed spatially heterogeneous interplay of HyWnt3 and HyDkk1/2/4-C allows explaining formation of a ring pattern of HyDkk1/2/4-C.

Recently, the astacin protease HAS-7 has been identified and analysed in Hydra [40]. Similar to Dkks, canonical Wnt signalling is antagonised on the protein level, but here, based on degradation rather than 
receptor isolation. Furthermore, there are several parallels between HyDkk1/2/4-C and HAS-7: both show similar expression patterns, are assumed to interact with canonical Wnt signalling via negative feedback loops and are additionally activated by factors downstream of secreted Wnt molecules. Thus, in the presented model, HyDkk1/2/4-C may be replaced or augmented by HAS-7.

\section{Submodel for tentacle formation}

The model describing head and body-axis formation was coupled to an additional subsystem describing tentacle formation based on the activator-inhibitor model. The tentacle system receives information from canonical Wnt signalling and the SD, but does not feed back to the body-axis formation subsystem $[13,26,73,74]$. Including the tentacle-formation process in the model provided data for additional model verification, e.g., accounting for ALP treatment. Notably, we assumed that the SD had a positive influence on the expression of tentacle-specific genes, whereas HyWnt3/ $\beta$-catenin inhibited the tentacle system. The assumed interactions among $\beta$-catenin/HyWnt3, the tentacles, and the SD could explain a broad range of experimental observations (presented in detail and discussed e.g. in Ref. $[26,28,41,44]$ ).

\section{Submodel for foot formation}

The model was completed by including a pattern-formation subsystem for the foot. Similar to the tentacle system, the foot system consists of a separate (phenomenological) activator-inhibitor model that interacts with the SD. More details, including experimental motivations, are given in [26, 28, 41]. Importantly, we do not assume any influence from the foot system on the SD, in order to exclude any influence from the foot system on the new proposed core axis formation system.

\section{Evolving geometry of the model}

To model the realistic geometry of the Hydra tissue bilayer, we adopted the mechano-chemical modelling approach proposed in Ref. [36,75] and extended it to describe small deformations of a thin tissue layer (similar to Ref. [35]). In particular, chemical reaction-diffusion equations were defined on a curved twodimensional (2D) surface embedded in a three-dimensional (3D) space. The geometry of the curved surface evolved over time following a gradient-flow of a Helfrich-type energy, reflecting the property that bending of the tissue away from a preferred local curvature is energetically unfavourable. In contrast to the fully coupled mechano-chemical model of [35], the current model did not consider any feedback from the mechanical properties of the tissue to the gene expression processes. Consequently, the mechanical part of the model did not control the pattern-formation mechanism based purely on molecular interactions but did provide a realistic description of the tissue as a radial-symmetric ellipsoid (or, in the case of aggregates, a sphere) that undergoes local deformations due to the gene expression patterns.

\section{Model equations and simulations}

\section{General structure of the mathematical model}

The above processes were translated into a mathematical model in terms of a system of partial differential equations (PDE). We applied a continuous modelling approach, which was justified by the large number of cells $\left(\geq 10^{4}\right)$ in the system [76]. The cell bilayer forming a hollow tissue ellipsoid was at any time $t$ approximated by a closed $2 \mathrm{D}$ surface $\Gamma(t)$, embedded in a $3 \mathrm{D}$ space. Hence, the different cell layers have not been modelled. The evolution of $\Gamma(t)$ is given by a diffeomorphic time-dependent representation $\vec{X}$, parameterised over the unit sphere $S^{2} \subset \mathbb{R}^{3}$. Thus, $\Gamma(t)$ is the image of $\vec{X}(\cdot, t)$ with $\vec{X}(\vec{s}, t): S^{2} \times[0, T] \rightarrow$ $\mathbb{R}^{3}$ for a $T \in \mathbb{R}_{>0}$. Local concentrations of different gene products $a$ at time $t$ are given by continuous functions $\Phi_{a}$ on the deforming tissue surface $\Gamma(t)$, defined as gene product concentrations per cell volume, $\Phi_{a}(t): \Gamma(t) \rightarrow \mathbb{R}_{\geq 0}$. The tissue evolution is coupled to chemical processes modelled in $S^{2}$ by identifying 


\begin{tabular}{|c|c|}
\hline Variable name & Explanation \\
\hline$W n t_{B}$ & $\begin{array}{l}\text { cell-local head-related molecule complex (e.g. comprising bounded HyWnt3, } \\
\text { nuclear } \beta \text {-catenin and Tcf) strongly related to formation of the oral pole in } \\
\text { Hydra (e.g., Ref. [11-13]). }\end{array}$ \\
\hline$D k k A$ & Dickkopf1/2/4-A - a HyWnt3/ $\beta$-catenin antagonist in Hydra $[14,15]$ \\
\hline$W n t_{F}$ & free (secreted/diffusing) HyWnt3 \\
\hline$D k k C$ & $\begin{array}{l}\text { Dickkopf1 } / 2 / 4-\mathrm{C} \text { - a HyWnt } 3 / \beta \text {-catenin antagonist in Hydra [15]. Optionally } \\
\text { replaced or augmented by the astacin protease HAS- } 7[40] \text {. }\end{array}$ \\
\hline$S D$ & $\begin{array}{l}\text { source density (SD) (also called e.g. "positional value" or "head forming } \\
\text { competence") - long-lasting information on the body-axis gradient; molecu- } \\
\text { lar/physical nature unknown. }\end{array}$ \\
\hline
\end{tabular}

Table 1. Model variables and their biological meanings.

material points $\vec{X}(\vec{s}, t)$ on $\Gamma(t)$ with $\vec{s} \in S^{2}$. The latter is ensured because $\vec{X}$ is smooth and bijective. Thus, for each $\vec{s} \in S^{2}, t \in[0, T]$, we defined the function $\phi_{a}: S^{2} \times[0, T] \rightarrow \mathbb{R}_{\geq 0}$ by $\phi_{a}(\vec{s}, t)=\Phi_{a}(\vec{X}(\vec{s}, t))$.

\section{Model of molecular interactions}

As above, the model accounted for the spatio-temporal dynamics of five different chemical components (Table 1). In particular, the model variable $W n t_{B}$ represents bounded HyWnt3, Tcf and $\beta$-catenin, which are responsible for head activation [11-13]. Wnt $t_{F}$ depicts free/secreted HyWnt3. DkkA and $D k k C$ describe HyDkk1/2/4-A and HyDkk1/2/4-C, respectively [14,15], and $S D$ describes the source density $[8,24,53,66,67]$. Finally, also the tentacle and the foot system have been simulated for completeness, however, they do not influence the core-patterning system presented here but are used for additional model validation (such as ALP treatment) only and are not in the focus of this study. More details on the equations for the foot respectively the tentacle system are e.g., given in Ref. [26, 28].

The resulting reaction-diffusion system defined on the domain corresponding to the tissue is given in Eq. (1)-(5). The process of spatial movement of the molecules is described by a surface LaplaceBeltrami operator $\Delta^{\Gamma}(\cdot)$. Eq. (1)-(5) describe the dynamics of the new axis-formation model based on the interplay among the HyWnt3/ $\beta$-catenin/Tcf-complex, the two HyDkk1/2/4 molecules, and the SD. We want to point out that this system can be even reduced to a system of 4 equations only, condensing cell-local and secreted Wnt to one single virtual component (c.f., Appendix). However, in this reduced system, the SD shows relatively fast dynamics, which may not correspond to the long-term behaviour of the experimentally observed SD readout.

In particular, Eq. (1) describes cell-local $\beta$-catenin/HyWnt3/Tcf dynamics, where HyWnt3 is bounded to receptors. This includes degradation, a small diffusion (mainly for numerical stability), and a production activated by the SD and inhibited by the two Dkks. Furthermore, the term $\left(1.0+0.1 W n t_{B}\right)$ in the denominator of the product term represents a saturation effect, i.e. an upper bound of gene expression that exists for each gene/promoter. Small diffusion has been added for numerical stability only. Eq. (2) describes the dynamics of HyDkk1/2/4-A, including diffusion (because Dkks are secreted), degradation, and a product that is reduced by the $\beta$-catenin/HyWnt3/Tcf-complex [14,15]. Eq. (3) describes freely diffusing HyWnt3 depending on the SD and the $\beta$-catenin/HyWnt3/Tcf complex, and Eq. (4) describes the dynamics of HyDkk1/2/4-C strongly related to those of HyDkk1/2/4-A (Eq. (3)) [15]. Finally, Eq. (5) represents the SD. For further details and motivations see Ref. [26, 28]. 


$$
\begin{aligned}
\partial_{t} W n t_{B}= & a_{1} \Delta^{\Gamma} W n t_{B}+\frac{b_{1} S D}{\left(0.1+0.1 D k k A^{2}\right)(1.0+0.3 D k k C)\left(1.0+0.1 W n t_{B}\right)}-c_{1} W n t_{B} \\
\partial_{t} D k k A= & a_{2} \Delta^{\Gamma} D k k A+\frac{b_{2}}{\left(0.1+0.1 W n t_{B}^{2}\right)}-c_{2} D k k A \\
\partial_{t} W n t_{F}= & a_{3} \Delta^{\Gamma} W n t_{F}+b_{3} S D\left(W n t_{B}\right)^{2}-c_{3} W n t_{F} \\
\partial_{t} D k k C= & a_{4} \Delta^{\Gamma} D k k C+\frac{b_{4} W n t_{F}}{\left(1.0+W n t_{B}\right)}-c_{4} D k k C \\
\partial_{t} S D= & a_{5} \Delta^{\Gamma} S D+b_{5} W n t_{B}+3 \times 10^{-4}-c_{5} S D
\end{aligned}
$$

\section{Model of tissue mechanics}

The chemical equations Eq. (1)-(9) are augmented by a set of equations representing the deforming tissue surface. Notably, we treated the tissue as purely elastic, and elastic tissue deformations were based on minimisation of the Helfrich free energy [77], which is given by

$$
\mathcal{F}_{\text {bend }}=\int_{\Gamma} \kappa\left(H-H_{0}\left(W n t_{B}, \text { Tent }, \text { Foot }\right)\right)^{2} d \vec{S} .
$$

Here, $H$ is the mean curvature, $\kappa$ the bending rigidity, and $H_{0}$ the spontaneous curvature $[36,75] . H_{0}$ represents the locally preferred tissue curvature, which again may depend on local morphogen concentrations. We assumed $H_{0}\left(W n t_{B}\right.$, Tent, Foot $)=1.5 W n t_{B}+3.0 F o o t+10.0 T e n t$, based on the observation of local tissue evaginations during head, foot, and tentacle formation [78-80]. Local area-conserving evolution of the deforming Hydra tissue was finally given by the $L^{2}$-gradient flow of the total energy including a local Lagrange multiplier. For further details, see Ref. [75].

\section{Numerical implementation}

The mathematical model was simulated using the finite element library Gascoigne [81], based on approximation of the fourth order PDEs in a mixed formulation. We applied linear finite elements for spatial discretisation, and a semi-implicit Euler scheme for time discretisation. For further details of the computation scheme, see $[36,75]$.

\section{Parameters and initial conditions}

For simulations of the unperturbed system, we applied the following parameters according to Ref. [26,28]): $a_{1}=18 \times 10^{-5}, b_{1}=15 \times 10^{-3}, c_{1}=3 \times 10^{-3}$,

$a_{2}=18 \times 10^{-7}, b_{2}=3 \times 10^{-3}, c_{2}=4 \times 10^{-3}$,

$a_{3}=22 \times 10^{-2}, b_{3}=3 \times 10^{-3}, c_{3}=4 \times 10^{-3}$,

$a_{4}=1 \times 10^{-7}, b_{4}=1 \times 10^{-2}, c_{4}=1 \times 10^{-2}$,

$a_{5}=11 \times 10^{-6}, b_{5}=3 \times 10^{-4}, d_{5}=3 \times 10^{-4}$,

To approximate the geometry of the Hydra tissue, initial conditions for $X_{1}, X_{2}$, and $X_{3}$ were parametrised over a closed 2D unit-sphere $S^{2}$ embedded in 3D space with $X_{1}(t=0) \equiv X_{2}(t=0) \equiv 0$ and $X_{3}(t=0)=4 \cdot s_{3}$, leading to a stretch in the direction of $s_{3}$ (given that $s_{1}, s_{2}, s_{3}$ are Eulerian coordinates of the $S^{2}$-surface). For all biological molecules, we used a stochastic initial distribution based on the standard random generator provided by $\mathrm{C}++$. Only the initial SD was modelled using an initial gradient given by $S D(t=0)=4.0 \cdot\left(\exp \left(s_{3}\right) / \exp (1)\right)$. For simulations of grafting an head regeneration (Fig. 3 $\mathrm{C}, \mathrm{D}), S D(t=0)=2.0 \cdot\left(\exp \left(s_{3}\right) / \exp (1)\right)$. has been used instead, to account for the fact that areas with 
maximal SD values have been removed with the head. Thus, in all simulations, only the geometric and chemical body-axis gradient were initially prescribed, corresponding to head-regeneration experiments. In simulations of ALP treatment, we modified the initial conditions for the source density by adding an offset by $S D(t=0)=2.0+4.0 \cdot\left(\exp \left(s_{3}\right) / \exp (1)\right)$. To simulate complete removal of HyDkk1/2/4-A respectively HyDkk1/2/4-A at a certain time point (after the head pattern had been established), the parameters $b_{2}$ and $b_{4}$ were set to zero. HyDkk1/2/4-A knockdown was modelled by increasing $c_{2}$ from $4 \times 10^{-3}$ to $6 \times 10^{-3}$. Hydra aggregates were simulated starting with a sphere as the initial geometry and using random initial conditions for all simulated molecules, including the SD.

\section{Acknowledgments}

This work is supported by Deutsche Forschungsgemeinschaft (DFG) under Germany's Excellence Strategy EXC-2181/1 - 390900948 (the Heidelberg STRUCTURES Excellence Cluster) and SFB1324 (B05 to A.MC and M.M., SFB1324/A05 to T.W.H, and SFB1324/B07 to S.Ö.).

\section{Appendix - a reduced version of the mutual inhibition model}

The proposed mutual inhibition model can be simplified to a four-component model exhibiting the same pattern formation mechanism. The model consists of reaction-diffusion equations

$$
\begin{aligned}
& \partial_{t} W n t=a_{1} \partial_{x x}^{2} W n t+\frac{b_{1} \frac{b_{5} W n t}{c_{5}}}{\left(0.1+0.1 D k k A^{2}\right)(1.0+0.3 D k k C)(1.0+0.1 W n t)}-c_{1} W n t ; \\
& \partial_{t} D k k A=a_{2} \partial_{x x}^{2} D k k A+\frac{b_{2}}{0.1+0.1 W n t^{2}}-c_{2} D k k A \\
& \partial_{t} S D=a_{3} \partial_{x x}^{2} S D+b_{3}\left(\frac{b_{5} W n t}{c_{5}}\right)(W n t)^{2}-c_{3} S D \\
& \partial_{t} D k k C=a_{4} \partial_{x x}^{2} D k k C+\frac{b_{4} S D}{1.0+W n t}-c_{4} D k k C
\end{aligned}
$$

with zero-flux voundary conditions. The model variables $W n t=W n t(x, t), D k k A=D k k A(x, t), S D=$ $S D(x, t)$, and $D k k C=D k k C(x, t)$ describe spatio-temporal distributions of the four molecules and the model parametres $a_{k}, b_{k}, c_{k}, k=1, \ldots, 5$ denote positive diffusion coefficients and reaction parameters, respectively. We consider a one-dimensional spatial domain $x \in(0, L)$.

Using this simplified model setting, we explore the pattern formation mechanism and check that the model exhibits Turing instability (diffusion-driven instability). Let us find parameter values, satisfying the conditions of Turing instability. We consider system (6), group reaction parameters and diffusion coefficients into the parameter vector $\theta$ and construct the objective function $f(\theta)$ by the following algorithm:

1. Consider system (6) without diffusion terms and find the spatially homogeneous steady state $w_{0}(\theta)$. This is done numerically by Newton's method.

2. Set $\sigma_{\max }=\max _{k=0, \ldots, 1000} \operatorname{Re}\left(\sigma_{1,2}^{k}\right)$, where $\sigma_{1,2}^{k}$ are eigenvalues of the linear operator $L(\theta)$, obtained by linearising spatially distributed system (6) around the steady state $w_{0}(\theta)$. Eigenvalues $\sigma_{1,2}^{k}$ correspond to the respective eigenvalues of the Laplace operator $-\frac{d^{2}}{d x^{2}}$ with Neumann boundary conditions, which are grouped in the increasing order.

3. If $\operatorname{Re}\left(\sigma_{1,2}^{0}\right)<0$ (system without the diffusion terms is linearly stable), then set $f(\theta)=\sigma_{\max }$. Else reject the current value $\theta$ by defining $f(\theta)=-1 e 15$.

Using the Differential Evolution algorithm [82], we perform heuristic search in the parameter space to find such parameter vector $\theta^{*}$ that $f\left(\theta^{*}\right)>0$. Next, we simulate the MOL discretisation of system 

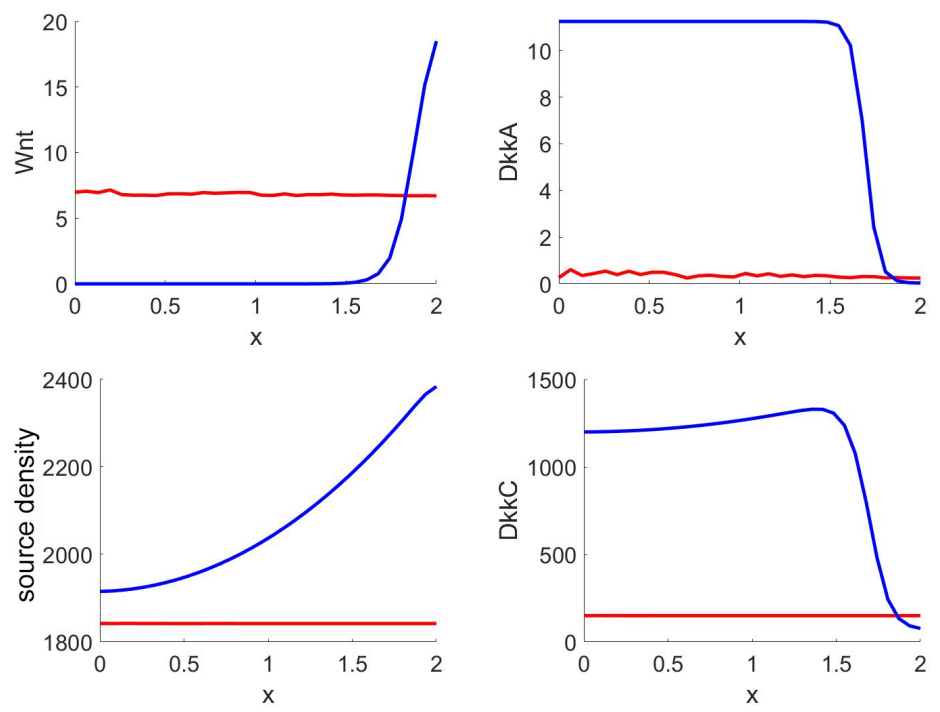

Figure 5. Pattern formation in model (6): initial data (red) and steady-state pattern (blue).

(6), taking linearly scaled small random perturbations of the homogeneous steady state $w_{0}(\theta)$ as initial data. A typical pattern is shown in Figure 5. We conduct all experiments in MATLAB, and perform the numerical integration of the equations by using the explicit Dormand-Prince method (built-in ode45 routine). Additionally, we verify our results by integrating the system with implicit TR-BDF2 (second order Trapezoidal Rule - Backward Differentiation Formula) method [83].

\section{References}

1. ten Berge D, Koole W, Fuerer C, Fish M, Eroglu E, et al. (2008) Wnt signaling mediates selforganization and axis formation in embryoid bodies. Cell stem cell 3: 508-518.

2. Petersen CP, Reddien PW (2009) Wnt signaling and the polarity of the primary body axis. Cell 139: $1056-68$.

3. Lewis SL, Khoo PL, De Young RA, Steiner K, Wilcock C, et al. (2008) Dkk1 and Wnt3 interact to control head morphogenesis in the mouse. Development 135: 1791-1801.

4. Kuang HB, Miao CL, Guo WX, Peng S, Cao YJ, et al. (2009) Dickkopf-1 enhances migration of HEK293 cell by beta-catenin/E-cadherin degradation. Frontiers in bioscience (Landmark edition) 14: $2212-2220$.

5. Ou L, Fang L, Tang H, Qiao H, Zhang X, et al. (2016) Dickkopf wnt signaling pathway inhibitor 1 regulates the differentiation of mouse embryonic stem cells in vitro and in vivo. Molecular medicine reports 13: $720-730$.

6. Niehrs C (2006) Function and biological roles of the Dickkopf family of Wnt modulators. Oncogene 25: $7469-81$.

7. Logan CY, Nusse R (2004) The Wnt signaling pathway in development and disease. Annu Rev Cell Dev Biol 20: 781-810. 
8. Grimmelikhuijzen C, Schaller H (1979) Hydra as a model organism for the study of morphogenesis. TIBS 4: 265-7.

9. Meinhardt H (2008) Models of biological pattern formation: from elementary steps to the organization of embryonic axes. Curr Top Dev Biol 81: 1-63.

10. Galliot B (2012) Hydra, a fruitful model system for 270 years. Int J Dev Biol 56: 411-23.

11. Hobmayer B, Rentzsch F, Kuhn K, Happel CM, von Laue CC, et al. (2000) Wnt signalling molecules act in axis formation in the diploblastic metazoan Hydra. Nature 407: 186-9.

12. Gee L, Hartig J, Law L, Wittlieb J, Khalturin K, et al. (2010) Beta-catenin plays a central role in setting up the head organiser in hydra. Dev Biol 340: 226-24.

13. Broun M, Gee L, Reinhardt B, Bode HR (2005) Formation of the head organizer in Hydra involves the canonical Wnt pathway. Development 132: 2907-16.

14. Guder C, Pinho S, Nacak TG, Schmidt HA, Hobmayer B, et al. (2006) An ancient Wnt-dickkopf antagonism in Hydra. Development 133: 901-11.

15. Augustin R, Franke A, Khalturin K, Kiko R, Siebert S, et al. (2006) Dickkopf related genes are components of the positional value gradient in Hydra. Dev Biol 296: 62-70.

16. Lengfeld T, Watanabe H, Simakov O, Lindgens D, Gee L, et al. (2009) Multiple Wnts are involved in Hydra organizer formation and regeneration. Dev Biol 330: 186-99.

17. Krupnik VE, Sharp JD, Jiang C, Robison K, Chickering TW, et al. (1999) Functional and structural diversity of the human Dickkopf gene family. Gene 238: 301-313.

18. Fedders H, Augustin R, Bosch TCG (2004) A dickkopf- 3-related gene is expressed in differentiating nematocytes in the basal metazoan hydra. Development genes and evolution 214: 72-80.

19. Gierer A, Berking S, Bode H, David C, Flick K, et al. (1972) Regeneration of hydra from reaggregated cells. Nature New Biol 239: 91: 98-101.

20. Technau U, Cramer von Laue C, Rentzsch F, Luft S, Hobmayer B, et al. (2000) Parameters of self-organization in Hydra aggregates. Proc Natl Acad Sci U S A 97: 12127-31.

21. Technau U, Holstein TW (1992) Cell sorting during the regeneration of Hydra from reaggregated cells. Dev Biol 151: 117-27.

22. Sato M, Tashiro H, Oikawa A, Sawada Y (1992) Patterning in hydra cell aggregates without the sorting of cells from different axial origins. Dev Biol 151: 111-6.

23. Turing AM (1953) The chemical basis of morphogenesis. Phil Trans R Soc Lond B 237: 37-72.

24. Gierer A, Meinhardt H (1972) A theory of biological pattern formation. Kybernetik 12: 30-9.

25. Meinhardt H, Gierer A (2000) Pattern formation by local self-activation and lateral inhibition. Bioessays 22: 753-60.

26. Meinhardt H (2012) Modeling pattern formation in hydra: A route to understanding essential steps in development. Int J Dev Biol 56: 447-62.

27. Hiscock TW, Megason SG (2015) Mathematically guided approaches to distinguish models of periodic patterning. Development 142: 409-19. 
28. Meinhardt H (1993) A model for pattern formation of hypostome, tentacles, and foot in hydra: how to form structures close to each other, how to form them at a distance. Dev Biol 157: 321-33.

29. Scholes NS, Schnoerr D, Isalan M, Stumpf MP (2019) A comprehensive network atlas reveals that turing patterns are common but not robust. Cell Sys 9: 243-57.

30. Diego X, Marcon L, Müller P, Sharpe J (2018) Key features of turing systems are determined purely by network topology. Phys Rev X 8: 021071.

31. Marciniak-Czochra A (2003) Receptor-based models for pattern formation in hydra. J Biol Syst 11: $293-324$.

32. Marciniak-Czochra A (2006) Receptor-based models with hysteresis for pattern formation in hydra. Math Biosci 199: 97-119.

33. S Härting AMC, Takagi I (2017) Stable patterns with jump discontinuityin systems with turing instability and hysteresis. Disc Cont Dyn Syst A 37: 757-800.

34. Veerman F, Mercker M, Marciniak-Czochra A (2021) Beyond turing: Far-from-equilibrium patterns and mechano-chemical feedback. bioRxiv doi: https://doi.org/10.1101/2021.03.10.434636.

35. Mercker M, Köthe A, Marciniak-Czochra A (2015) Mechanochemical symmetry breaking in Hydra aggregates. Biophys J 108: 2396-407.

36. Mercker M, Hartmann D, Marciniak-Czochra A (2013) A mechanochemical model for embryonic pattern formation: coupling tissue mechanics and morphogen expression. PLoS One 8: e82617.

37. Brinkmann F, Mercker M, Richter T, Marciniak-Czochra A (2018) Post-Turing tissue pattern formation: Advent of mechanochemistry. PLoS Comput Biol 14: e1006259.

38. Mercker M, Brinkmann F, Marciniak-Czochra A, Richter T (2016) Beyond Turing: mechanochemical pattern formation in biological tissues. Biol Direct 11: 1-15.

39. Pietak A, Levin M (2017) Bioelectric gene and reaction networks: computational modelling of genetic, biochemical and bioelectrical dynamics in pattern regulation. J R Soc Interface 14: 20170425.

40. Ziegler B, Yiallouros I, Trageser B, Kumar S, Mercker M, et al. (2021) A Wnt-specific astacin proteinase controls head formation in Hydra. BMC Biol 19: doi: 10.1186/s12915-021-01046-9.

41. Meinhardt H (2012) Turing's theory of morphogenesis of 1952 and the subsequent discovery of the crucial role of local self-enhancement and long-range inhibition. Interface Focus 2: 407-16.

42. Vogg MC, Beccari L, Iglesias Oll Rampon C, Vriz S, et al. (2019) An evolutionarily-conserved Wnt3/ $\beta$-catenin/Sp5 feedback loop restricts head organizer activity in Hydra. Nat Commun 10: 312.

43. Huggins IJ, Bos T, Gaylord O, Jessen C, Lonquich B, et al. (2017) The WNT target SP5 negatively regulates WNT transcriptional programs in human pluripotent stem cells. Nat Commun 8: doi: 10.1038/s41467-017-01203-1.

44. Mercker M, Lengfeld T, Höger S, Tursch A, Lommel M, et al. (2021) $\beta$-catenin and canonical wnts control two separate pattern formation systems in hydra: Insights from mathematical modelling. BioRxiv : doi: https://doi.org/10.1101/2021.02.05.429954. 
45. Lommel M, Strompen J, Hellewell AL, Balasubramanian GP, Christofidou ED, et al. (2018) Hydra mesoglea proteome identifies thrombospondin as a conserved component active in head organizer restriction. Sci Rep 8: 11753.

46. Iachetta R, Ambrosone A, Klimovich A, Wittlieb J, Onorato G, et al. (2018) Real time dynamics of $\beta$-catenin expression during Hydra development, regeneration and wnt signalling activation. Int J Dev Biol 62: 311-8.

47. Briscoe J, Small S (2015) Morphogen rules: design principles of gradient-mediated embryo patterning. Development 142: 3996-4009.

48. Sacks PG, Davis LE (1979) Production of nerveless Hydra attenuata by hydroxyurea treatments. J Cell Sci 37: 189-203.

49. Gufler S, Artes B, Bielen H, Krainer I, Eder MK, et al. (2018) $\beta$-Catenin acts in a positionindependent regeneration response in the simple eumetazoan Hydra. Dev Biol 433: 310-23.

50. Petersen HO, Höger SK, Looso M, Lengfeld T, Kuhn A, et al. (2015) A comprehensive transcriptomic and proteomic analysis of Hydra head regeneration. Mol Biol Evol 32: 1928-47.

51. Tursch A, Bartsch N, Holstein TW (2020) MAPK signaling links the injury response to Wnt-regulated patterning in Hydra regeneration. Preprint, BioRxiv, doi: https://doiorg/101101/20200706189795 .

52. Browne EN (1909) The production of new hydranths in Hydra by the insertion of small grafts. J Exp Zool 7: 1-23.

53. MacWilliams HK (1983) Hydra transplantation phenomena and the mechanism of hydra head regeneration. II. Properties of the head activation. Dev Biol 96: 239-57.

54. MacWilliams HK (1983) Hydra transplantation phenomena and the mechanism of hydra head regeneration. I. Properties of the head inhibition. Dev Biol 96: 217-38.

55. Campbell RD (1976) Elimination by Hydra interstitial and nerve cells by means of colchicine. J Cell Sci 21: 1-13.

56. Marcum BA, Campbell RD (1978) Development of Hydra lacking nerve and interstitial cells. J Cell Sci 29: 17-33.

57. Sugiyama T, Fujisawa T (1978) Genetic analysis of developmental mechanisms in hydra. V. Cell lineage and development of chimera hydra. J Cell Sci 32: 215-32.

58. Galliot B, Buzgariu W, Schenkelaars Q, Wenger Y (2018) Non-developmental dimensions of adult regeneration in Hydra. Int J Dev Biol 62: 373-81.

59. Wenger Y, Buzgariu W, Galliot B (2016) Loss of neurogenesis in Hydra leads to compensatory regulation of neurogenic and neurotransmission genes in epithelial cells. Phil Trans R Soc Lond B Biol Sci 371: 20150040.

60. Nakamura Y, Tsiairis CD, Özbek S, Holstein TW (2011) Autoregulatory and repressive inputs localize Hydra Wnt3 to the head organizer. Proc Natl Acad Sci U S A 108: 9137-42.

61. Murray J (2003) Mathematical Biology II: Spatial models and biomedical applications. New york: Springer-Verlag. 
62. Plaza R, Sanchez-Garduno F, Padilla P, Barrio R, Maini P (2004) The effect of growth and curvature on pattern formation. Journal of Dynamics and Differential Equations : 1093-1121.

63. Bode H (2011) Axis formation in Hydra. Annu Rev Genet 45: 105-17.

64. Shimizu H (2012) Transplantation analysis of developmental mechanisms in Hydra. Int J Dev Biol 56: $463-72$.

65. Broun M, Bode HR (2002) Characterization of the head organizer in Hydra. Development 129: $875-84$.

66. Hassel M, Bieller A (1996) Stepwise transfer from high to low lithium concentrations increases the head-forming potential in Hydra vulgaris and possibly activates the PI cycle. Dev Biol 177: $439-48$.

67. Wolpert L, Clarke MR, Hornbruch A (1972) Positional signalling along Hydra. Nature 239: 101-5.

68. Bode HR (2003) Head regeneration in Hydra. Dev Dyn 226: 225-36.

69. Hassel M, Albert K, Hofheinz S (1993) Pattern formation in Hydra vulgaris is controlled by lithiumsensitive processes. Developmental biology 156: 362-71.

70. Niida A, Hiroko T, Kasai M, Furukawa Y, Nakamura Y, et al. (2004) DKK1, a negative regulator of wnt signaling, is a target of the beta-catenin/TCF pathway. Oncogene 23: 8520-8526.

71. Gonzalez-Sancho JM, Aguilera O, GarcM, PendFranco N, Pe et al. (2005) The Wnt antagonist DICKKOPF-1 gene is a downstream target of beta-catenin/TCF and is downregulated in human colon cancer. Oncogene 24: 1098-1103.

72. Bazzi H, Fantauzzo KA, Richardson GD, Jahoda CAB, Christiano AM (2007) The Wnt inhibitor, Dickkopf 4, is induced by canonical Wnt signaling during ectodermal appendage morphogenesis. Dev Biol 305: 498-507.

73. Technau U, Holstein TW (1995) Head formation in Hydra is different at apical and basal levels. Development 121: 1273-82.

74. Smith KM, Gee L, Bode HR (2000) HyAlx, an aristaless-related gene, is involved in tentacle formation in hydra. Development 127: 4743-52.

75. Mercker M, Marciniak-Czochra A, Richter T, Hartmann D (2013) Modeling and computing of deformation dynamics of inhomogeneous biological surfaces. SIAM Journal on Applied Mathematics 73(5): 1768-1792.

76. Soriano J, Rüdiger S, Pullarkat P, Ott A (2009) Mechanogenetic coupling of Hydra symmetry breaking and driven Turing instability model. Biophys J 96: 1649-60.

77. Helfrich W (1973) Elastic properties of lipid bilayers: theory and possible experiments. Z Naturforsch [C] 28: 693-703.

78. Philipp I, Aufschnaiter R, Özbek S, Pontasch S, Jenewein M, et al. (2009) Wnt/beta-catenin and noncanonical Wnt signaling interact in tissue evagination in the simple eumetazoan Hydra. Proc Natl Acad Sci U S A 106: 4290-5.

79. Aufschnaiter R, Wedlich-Söldner R, Zhang X, Hobmayer B (2017) Apical and basal epitheliomuscular F-actin dynamics during bud evagination. Biol Open 6: 1137-48. 
80. Amimoto Y, Kodama R, Kobayakawa Y (2006) Foot formation in hydra: a novel gene, anklet, is involved in basal disk formation. Mechanisms of development 123: 352-361.

81. Becker R, Braack M, Dunne T, Meidner D, Richter T, et al. (2005) Gascoigne 3D- a finite element toolbox (http://www.gascoigne.uni-hd.de).

82. Storn R, Price K (1997) Differential Evolution - a simple and efficient heuristic for global optimization over continuous spaces. Journal of Global Optimization 11: 341-359.

83. Bank R, Coughran W, Fichtner W, Grosse E, Rose D, et al. (1985) Transient simulation of silicon devices and circuits. IEEE Transactions on Computer-Aided Design of Integrated Circuits and Systems 4: 436-451. 